\title{
Sleep apnea detection from a single-lead ECG signal with automatic feature-extraction through a modified LeNet-5 convolutional neural network
}

\author{
Tao Wang ${ }^{\text {Corresp., } 1}$, Changhua Lu ${ }^{1,2}$, Guohao Shen ${ }^{1}$, Feng Hong ${ }^{1}$ \\ ${ }^{1}$ School of Computer and Information, Hefei University of Technology, Hefei, Anhui, China \\ 2 School of Software, Hefei University of Technology, Hefei, Anhui, China \\ Corresponding Author: Tao Wang \\ Email address: wtustc@mail.ustc.edu.cn
}

Sleep apnea (SA) is the most common respiratory sleep disorder, leading to some serious neurological and cardiovascular diseases if left untreated. The diagnosis of SA is traditionally made using Polysomnography (PSG). However, this method requires many electrodes and wires, as well as an expert to monitor the test. Several researchers have proposed instead using a single channel signal for SA diagnosis. Among these options, the ECG signal is one of the most physiologically relevant signals of SA occurrence, and one that can be easily recorded using a wearable device. However, existing ECG signal-based methods mainly use features (i.e. frequency domain, time domain, and other nonlinear features) acquired from ECG and its derived signals in order to construct the model. This requires researchers to have rich experience in ECG, which is not common. A convolutional neural network (CNN) is a kind of deep neural network that can automatically learn effective feature representation from training data and has been successfully applied in many fields. Meanwhile, most studies have not considered the impact of adjacent segments on SA detection. Therefore, in this study, we propose a modified LeNet-5 convolutional neural network with adjacent segments for SA detection. Our experimental results show that our proposed method is useful for SA detection, and achieves better or comparable results when compared with traditional machine learning methods. 
1 Sleep apnea detection from a single-lead ECG signal

2 with automatic feature-extraction through a modified

3 LeNet-5 convolutional neural network

4

5 Tao Wang ${ }^{1}$, Changhua $\mathrm{Lu}^{1,2}$, Guohao Shen ${ }^{1}$, Feng Hong ${ }^{1}$

6

$7{ }^{1}$ School of Computer and Information, Hefei University of Technology, Hefei, Anhui, China

$8{ }^{2}$ School of Software, Hefei University of Technology, Hefei, Anhui, China

9

10 Corresponding Author:

11 Tao Wang ${ }^{1}$

12 Fuicui Lake Campus of HeFei University of Technology, Hefei, Anhui, China

13 Email address: wtustc@mail.ustc.edu.cn 


\section{Abstract}

16 Sleep apnea (SA) is the most common respiratory sleep disorder, leading to some serious 17 neurological and cardiovascular diseases if left untreated. The diagnosis of SA is traditionally 18 made using Polysomnography (PSG). However, this method requires many electrodes and wires, 19 as well as an expert to monitor the test. Several researchers have proposed instead using a single 20 channel signal for SA diagnosis. Among these options, the ECG signal is one of the most 21 physiologically relevant signals of SA occurrence, and one that can be easily recorded using a wearable device. However, existing ECG signal-based methods mainly use features (i.e. frequency domain, time domain, and other nonlinear features) acquired from ECG and its derived signals in order to construct the model. This requires researchers to have rich experience in ECG, which is not common. A convolutional neural network $(\mathrm{CNN})$ is a kind of deep neural network that can automatically learn effective feature representation from training data and has been successfully applied in many fields. Meanwhile, most studies have not considered the impact of adjacent segments on SA detection. Therefore, in this study, we propose a modified LeNet-5 convolutional neural network with adjacent segments for SA detection. Our experimental results show that our proposed method is useful for SA detection, and achieves better or comparable results when compared with traditional machine learning methods.

Keywords: Sleep Apnea, ECG, LeNet-5, Convolutional Neural Network, Automatic FeatureExtraction 


\section{Introduction}

Sleep apnea (SA) is the most common respiratory disorder, caused by partial or complete obstructions of the upper respiratory tract (Li et al. 2018; Punjabi 2008). During sleep, SA events can occur hundreds of times, and, if repeated over a long period of time, can cause serious neurological and cardiovascular complications such as memory loss, high blood pressure, congestive heart failure, and poor cognitive ability during the day (Khandoker et al. 2009; Sharma \& Sharma 2016; Varon et al. 2015; Young et al. 1997). Reportedly, approximately 5\% of women and $14 \%$ of men have SA syndrome in the United States, and the incidence of the disease is increasing in various populations (Peppard et al. 2013; Song et al. 2016). The severity of SA is clinically assessed using the apnea-hypopnea index (AHI). Subjects with an $A H I>5$ combined with other symptoms (i.e. excessive sleepiness and poor cognitive ability during the day) are diagnosed with SA (Marcus et al. 2012; Song et al. 2016).

Polysomnography (PSG) is one of the most common tests used for SA diagnosis. It analyzes physiological signals (e.g. airflow, electroencephalogram (EEG), electrocardiogram (ECG), and respiratory signals) during sleep (Bloch 1997; Song et al. 2016) in a hospital, and requires the patient to wear a number of electrodes and wires while an expert monitors the whole examination process. This complicated and uncomfortable examination experience has limited the application of PSG in clinical practice. To this end, several methods using a single channel signal (i.e. ECG (Penzel et al. 2003), $\mathrm{SaQ}_{2}$ (Hornero et al. 2007), and respiratory sound (Azarbarzin \& Moussavi 2013)) for SA diagnosis have been proposed (Song et al. 2016) to reduce costs and to be more easily implemented. Among these, using an ECG signal has been the most popular method because it is one of the most physiologically relevant signals of SA occurrence and can be easily recorded using a wearable device.

For example, Song et al. developed a Hidden Markov Model (HMM) SA detection method using the frequency domain and time domain features extracted from EDR signals and ECG signals, and their model achieved an accuracy of $86.2 \%$ in per-segment SA detection (Song et al. 2016). Sharam et al. proposed an RBF kernel LS-SVM for the per-segment SA detection based on features extracted from RR intervals by the hermit basic function, and the accuracy of their model was 83.8\% (Sharma \& Sharma 2016). Existing methods mainly use frequency domain, time domain, and some nonlinear features acquired from ECG and its derived signals to construct the model. This requires researchers to have a wealth of relevant domain knowledge and experience, researchers with sufficient experience are uncommon. Recently, Li et al. proposed an SA detection method that uses stacked SAE to automatically extract features (Li et al. 2018). Their method avoids over-reliance on ECG domain knowledge, and achieved an accuracy of $84.7 \%$ in persegment classification. However, stacked SAE is essentially an unsupervised feature 
transformation that cannot extract features effectively (Kang et al. 2017). A convolutional neural network $(\mathrm{CNN})$ is a deep neural network that simulates the deep hierarchal structure of human vision (Matsugu et al. 2003). Compared to traditional machine learning methods, a CNN does not require hand-crafted features, and can automatically extract effective features through hierarchical layers. It has been successfully applied in speech recognition (AbdelHamid et al. 2012; Palaz \& Collobert 2015), image classification (Sharif Razavian et al. 2014; Wei et al. 2016), signal analysis (Kwon et al. 2018; Sedighi et al. 2015) and other fields. LeNet-5 is one CNN implementation with relatively few parameters and good performance (El-Sawy et al. 2016; LeCun 2015; Wen et al. 2018). It is worth noting that in a CNN, many parameters are prone to overfitting when training small data (i.e. the data used in this study), increasing the difficulty of this task. Therefore, the main objective of this study is to detect SA by automatically extracting features from RR intervals and amplitudes using LeNet-5. Previous studies (De Chazal et al. 2000; Maier et al. 2000; Yadollahi \& Moussavi 2009) have shown that adjacent segments offer useful information for SA detection. Additionally, we combine adjacent segments into our proposed method. Experimental results in the PhysioNet Apnea-ECG and UCD datasets show that our proposed method is robust, and its performance has been improved further since, promoting the clinical application of a single-lead ECG SA detection method.

\section{Materials \& Methods}

\section{Datasets}

To ensure reliable results, two separate datasets were used in this study. A brief description of the two datasets is provided below.

\section{PhysioNet Apnea-ECG dataset}

The first dataset was the PhysioNet Apnea-ECG dataset provided by Philipps University (Goldberger et al. 2000; Penzel et al. 2000). It contains a total of 70 single-lead ECG signal recordings (released set: 35 recordings, withheld set: 35 recordings), which were sampled at 100 $\mathrm{Hz}$ and ranged between 401 and 587 minutes. For each 1-minute ECG signal recording segment, the dataset provided an expert annotation (if there was an apnea event within this minute, it was labeled as SA; otherwise, normal). It was notable that there was no difference between hypopnea and apnea in the provided annotation file, and all events were either obstructive or mixed (central was not included). Additionally, these recordings were classified as Class A, Class B and Class C according to the Apnea-Hypopnea Index (AHI) value. Class A meant that the recording contained 10 or more SA segments per hour $(A H I \geq 10)$ and the entire recording had at least 100 SA segments. Class B meant that the recording included five or more SA segments per hour $(A H I \geq 5)$ and the 
104

entire recording contained five to $99 \mathrm{SA}$ segments. Class $\mathrm{C}$ (or Normal) meant that the recording had less than five segments of SA per hour $(A H I<5)$.

\section{UCD dataset}

The UCD dataset was the second dataset, which was collected by the University College Dublin, and can be downloaded from the PhysioNet website (https://physionet.org/physiobank/database/ucddb/). This dataset recorded the complete overnight PSG recordings of 25 (4 females and 21 males) suspected sleep disordered breathing patients, each contained 5.9 to 7.7 hours of ECG signal as well as an annotation of the start time and the duration of every apnea/hypopnea event. Considering that this study primarily performed SA detection on 1-minute ECG signal segments, we converted continuous ECG data to 1-minute intervals which we correlated with annotations for normal and apnea events. According to the definition of apnea, an event should last at least 10s. However, an apnea event lasting 10 s may be separated over two adjacent minutes, each having a smaller amount of apnea event time (Mostafa et al. 2018; Xie \& Minn 2012). In the case of apnea or hypopnea lasting 5 or more consecutive seconds, the minute is considered to be an apnea. Additionally, each recording was classified as Class A, Class B or Class $\mathrm{C}$ by the Apnea-Hypopnea Index (AHI) value.

\section{Preprocessing}

A method for automatically extracting features from RR intervals and amplitudes was developed in this study, and a preprocessing scheme was needed in order to obtain the RR intervals and amplitudes. Since several studies (De Chazal et al. 2000; Maier et al. 2000; Yadollahi \& Moussavi 2009) have shown that adjacent segment information is helpful for per-segment SA detection, the labeled segment and its surrounding \pm 2 segments of the ECG signal (five 1-minute segments in total) were all extracted for processing. We first used the Hamilton algorithm (Hamilton 2002) to find the R-peaks, then used the position of the R-peaks to calculate the RR intervals (distance between R-peaks) and extract the values of the R-peaks (amplitudes). Considering that the extracted RR intervals had some physiologically uninterpretable points, the median filter proposed by Chen et al. (Chen et al. 2015) was employed. Since the obtained RR intervals and amplitudes were not equal time intervals, which was required by our proposed method, cubic interpolation was further employed, and 900 points of RR intervals and 900 points of amplitudes over 5-minute segments were obtained. The detailed preprocessing scheme is shown in Figure 1.

\section{Convolutional Neural Network}

In recent years, a CNN has been used as a research hotspot in the field of artificial intelligence (AI). It is a deep neural network method that simulates the deep hierarchal structure of human 
137 vision and has been successfully applied in image classification, natural language processing 138 (NLP) and speech recognition (Palaz \& Collobert 2015; Sharif Razavian et al. 2014; Yin et al. 139 2017). Due to its proficiency in automatic feature extraction, $C N N$ is also used to design advanced 140 signal analysis methods (Kwon et al. 2018; Sedighi et al. 2015). For example, (Kiranyaz et al. 141 2015) used $\mathrm{CNN}$ for ECG classification. Here, we used a simple and effective CNN 142 implementation, LeNet-5, to construct our SA detection model. In the following section, we will 143 introduce both the standard LeNet-5 and our modified LeNet-5.

Architecture of the standard LeNet-5

The standard LeNet-5 proposed by LeCun et al. (LeCun 2015) was designed to solve the problem of character recognition. It consisted of an input layer, two convolution layers, two fully connected layers, two pooling layers and an output layer -- in total, seven layers. The details of each layer are described in (LeCun 2015). Formally, a set of $N$ images $\left\{X_{i}, y_{i}\right\}_{i=1}^{N}$ are taken, where $X_{i}$ is the original image data and $y_{i}$ is a class category of the image (i.e. 0 and 1). The difference between the predicted label $\hat{y}_{i}$ and the real label $y_{i}$ is calculated using the categorical cross entropy function, defined as follows:

$$
J(\omega, b) \triangleq-\frac{1}{N} \sum_{l=1}^{N} y_{l 1} \log \widehat{y_{l 1}}+\cdots+y_{l K} \log \widehat{y_{l K}}
$$

where $\omega$ and $b$ represent the weights and biases of the standard LeNet-5 network layers, respectively. $K$ is the number of class category and $\widehat{y_{l k}}$ corresponds to the softmax value of the $k^{\prime} t h$ class category, defined as:

$$
\widehat{y_{l k}}=\operatorname{softmax}\left(z_{k}\right)=\frac{e^{z_{k}}}{\sum_{i=1}^{K} e^{z_{i}}}
$$

where $z_{i}$ is the result of the corresponding $i$ 'th class category of the last fully connected output. The weight and bias parameters of the convolutional operation and fully connected layers were learned by back-propagating (BP) the derivative of the loss with respect to parameters throughout the entire network (Zeiler \& Fergus 2014).

Architecture of our modified LeNet-5

Unlike with character recognition, the time series used in this study had one-dimensional data, which is significantly different from two-dimensional character recognition problems. When 
164

165

166

167

168

169

170

171

172

173

174

175

176

177

178

179

180

181

182

183

184

185

186

187

188

189

190

191

192

193

194

195

compared with the millions of training samples in the field of character or image classification, the data samples used in this study were smaller, which increases the risk of overfitting. Moreover, SA detection is a binary classification problem that differs from character recognition. The feature maps, convolution layer strides and fully-connected layer nodes in the standard LeNet-5 may not be suitable for this scene. Therefore, we adjusted LeNet-5 as follows: 1) using a one-dimensional convolution operation instead of a two-dimensional convolution operation to feature extraction (Kiranyaz et al. 2015) ; 2) adding a dropout layer between the convolution layer and fully connected layer to avoid over-fitting (Srivastava et al. 2014); 3) retaining only one fully connected layer to reduce network complexity (Ma et al. 2018); 4) modifying the size of the convolution layer strides and the number of fully-connected layer nodes. The architecture and details of our modified LeNet-5 are shown in Figure 2 and Table 1, respectively. Compared to the standard LeNet-5, all convolution layer strides of our modified LeNet-5 were changed to two, and the number of feature maps was increased layer by layer. In particular, a dropout layer with a drop rate of 0.8 was added between the convolution layer and the fully connected layer, and the number of output layer nodes was reduced from 10 to two for our binary classification problem.

\section{Experiment settings}

In the field of SA detection based on a single-lead ECG signal, existing methods mainly extract suitable features based on expert experience, and then construct a model using the extracted features (Sharma \& Sharma 2016; Song et al. 2016; Varon et al. 2015), a process called feature engineering. In order to evaluate the performance of our proposed method, several popular feature engineering-based machine learning methods, including Support Vector Machine (SVM), KNearest Neighbor (KNN), Logistic Regression (LR) and Multi-Layer Perception (MLP), were employed for comparison. Various features that might have provided useful information for SA detection had been built in previous studies, and here we employed the features (RR intervals: 12 features, amplitudes: six features) that had an important effect on SA detection (De Chazal et al. 2000; Song et al. 2016) as the input of feature engineering-based methods. Table 2 lists the details of these features. Since some methods are sensitive to feature scales (i.e. KNN), the min-max normalization was used to normalize all features, which is defined as follows:

$$
x^{*}=\frac{x-x_{\min }}{x-x_{\max }}
$$

where $x$ is the feature to be normalized, and $x_{\max }$ and $x_{\min }$ are the maximum and minimum value in the features, respectively.

\section{Evaluation}


196

197

198

199

200

By following (Song et al. 2016; Varon et al. 2015), the specificity (Sp), sensitivity (Sn), accuracy (Acc) and area under the curve (AUC) were employed to evaluate the performance of our proposed method, defined as follows:

$$
\begin{aligned}
& \text { specificity }=\frac{T N}{T N+F P} \\
& \text { sensitivity }=\frac{T P}{T P+F N}
\end{aligned}
$$

$$
\text { accuracy }=\frac{T P+T N}{T P+T N+F P+F N}
$$

where $F P$ and TP stand for "false positive" and "true positive", respectively. $F N$ and $T N$ represent "false negative" and "true negative", respectively.

\section{Results \& Discussion}

In this study, two separate datasets were used to validate our proposed method. The PhysioNet Apnea-ECG dataset was used as benchmark data to evaluate our proposed method's performance. The UCD dataset is an independent dataset we used to check the robustness of our proposed method against other datasets.

\section{Per-segment SA detection}

Accurately predicting the presence of SA by given ECG segment (minute-by-minute) is key in this field, as it provides a solid foundation for the diagnosis of suspected SA patients. Therefore, we compared our proposed method with traditional machine learning methods on per-segment SA detection. The overall performance of the withhold set, including its specificity, sensitivity, accuracy and AUC, was used for comparison, as displayed in Table 3. As can be seen from Table 3 , our modified LeNet-5 with automatic feature extraction performed well in all measurements with a specificity of $90.3 \%$, sensitivity of $83.1 \%$, accuracy of $87.6 \%$ and AUC of 0.950 . Compared with the SVM that had the second highest accuracy, the overall performances were better by $6.0 \%$, $6.2 \%, 6.2 \%$ and 0.063 , respectively. It can also be seen from the results that KNN had the lowest prediction accuracy among the five methods, probably because the features extracted from the ECG signal were less spatially correlated and were not suitable for this scene, similar to the findings in literature (Sharma \& Sharma 2016). In summary, in per-segment SA detection, our proposed LeNet-5 with automatic feature extraction performed better than the commonly used feature engineering method.

\section{Per-recording classification}


225 A recording consists of multiple one-minute ECG segments, and the classification of each 226 recording refers to the overall SA diagnosis of these one-minute ECG segments, which is different 227 from per-segment SA detection. Clinically, AHI is used to distinguish SA from normal recordings. 228 Specifically, if the recording AHI is greater than 5, it is diagnosed as SA; otherwise it is considered 229 to be normal. The recording AHI is calculated using the results of per-segment SA detection, which 230 is defined as follows:

$$
A H I=\frac{60}{T} * \text { num of OSA segments }
$$

232

233

Where $T$ denotes the number of one-minute ECG segment signals, and $T / 60$ is the hour of the entire recording. Therefore, AHI is employed here to diagnose the recording SA, and the abovementioned measurement's accuracy, sensitivity, specificity and AUC are computed on the withheld set as listed in Table 4. It should be noted that the withheld set provided by the PhysioNet Apnea-ECG dataset had only 35 recordings, which may have resulted in low-precision persegment methods showing better per-recording performance. By following previous studies (Sharma \& Sharma 2016; Song et al. 2016), the correlation value between the experimentally determined $A H I$ and the actual $A H I$ were also adopted to ensure the reliability of the comparison. As shown in Table 4, when compared with SVM, LR, KNN and MLP, our modified LeNet-5 with an accuracy of $97.1 \%$, sensitivity of $100 \%$, specificity of $91.7 \%$ and AUC of 0.996 performed better in per-recording classification. The correlation value of our modified LeNet-5 further confirmed this result, which increased by 0.091 when compared to the second highest SVM method.

\section{Effect of automatic feature extraction}

In the previous parts, we discussed the overall performance of our proposed LeNet-5 in perrecording classification and per-segment detection. The results showed that, when compared with the existing methods, our proposed method significantly improved the performance in both perrecording classification and per-segment SA detection. Here, we will verify the power of the automatic feature extraction of our proposed method. Figure 3 displays the receiver operating characteristic (ROC) curves of our modified LeNet-5 and MLP in per-segment SA detection, since our modified LeNet-5 can be seen as a combination of convolutional neural networks $(\mathrm{CNN})$ for feature extraction and full connection (FC, also known as MLP) as classifier (Bae et al. 1998; Ludermir et al. 2006), meaning that the effects of the automatic extraction features obtained by our proposed LeNet-5 and the features extracted by traditional feature engineering can be directly compared. As shown in Figure 3, the LeNet-5's ROC curve is always above the MLP's ROC curve. These results suggest that the effect of the features extracted by our proposed automatic 
258 feature extraction method easily exceeded traditional feature engineering. Additionally, the 259 measurements of the two methods in Table 3 also verify this result.

\section{Robustness Evaluation}

261

262

263

264

265

266

267

268

269

270

271

272

273

274

275

276

277

278

279

280

281

282

283

284

285

286

287

288

289

290

Ten-fold cross-validation

Validating a method with a single small-size test dataset may be biased or lead to incorrect results (Sharma \& Sharma 2016; Song et al. 2016). To this end, we used ten-fold cross-validation to ensure that our proposed method was robust under different test datasets. The whole dataset (70 recordings) was randomly split into 10 groups, of which nine were adopted to train the classifiers (SVM, LR, KNN, MLP and LeNet-5), and the remaining one was used for the test, taken 10 times. The accuracy of the per-segment SA detection calculated on 10 different test groups was drawn and is shown in Figure 4. As seen in Figure 4, the accuracies obtained using the SVM, LR, KNN, MLP and LeNet-5 ranged from $71.9 \%$ to $88.6 \%$ (mean \pm standard deviation, $81.1 \% \pm 5.50 \%$ ), $71.7 \%$ to $87.8 \%$ (mean \pm standard deviation, $80.6 \% \pm 5.47 \%$ ), $72.5 \%$ to $84.8 \%$ (mean \pm standard deviation, $79.3 \% \pm 4.53 \%$ ), $75.4 \%$ to $89.9 \%$ (mean \pm standard deviation, $81.9 \% \pm 4.98 \%$ ) and $84.2 \%$ to $93.7 \%$ (mean \pm standard deviation, $88.7 \% \pm 3.05 \%$ ), respectively. These results suggest that our proposed LeNet-5 with automatic feature extraction was more robust, and could achieve consistent and significantly better performances in different test datasets.

\section{Validation on UCD database}

To ensure that our proposed method was robust in other datasets, we tested the performance of our modified LeNet-5 on an independent UCD dataset. Similar to the PhysioNet Apnea-ECG dataset, the dataset was divided into two parts, one for training and the other for verification. It is noteworthy that the original UCD dataset is continuously annotated based on the occurrence of events, which is different from the PhysioNet Apnea-ECG dataset, and we followed (Mostafa et al. 2018; Xie \& Minn 2012) in converting them to 1-minute interval annotations. Table 5 shows the performance of our modified LeNet-5 and traditional machine learning methods in per-segment SA detection and per-recording classification. As shown in Table 5, the overall performance of different methods on the UCD dataset was worse than that of the PhysioNet dataset, caused by the small number of SA annotations on the UCD dataset. However, our modified LeNet-5 still had better or comparable performance to the traditional machine learning methods. For example, when compared with SVM in per-segment SA detection, the accuracy of our modified LeNet-5 was $1.2 \%$ better. In per-recording classification, our modified LeNet-5 had the same accuracy as SVM, but the correlation increased by 0.373 . In general, our modified LeNet-5 is useful for SA detection.

\section{Comparison with existing works}


291 So far, several works on SA detection based on a single-lead ECG signal have been published in 292 the literature, and these works are mainly focused on feature engineering. Here, we compared our 293 proposed method with relevant work that used both withheld sets and released sets of the 294 PhysioNet Apnea-ECG dataset. However, a direct comparison was not available, due to the 295 different samples sizes ( $\mathrm{Li}$ et al. 2018). Table 6 shows the relevant work and performance of using 296 the same dataset for per-segment detection. The released set was used for training, and the withheld set was used for validation. As shown, the classification accuracy of existing works ranged from $83.4 \%$ to $86.2 \%$, which is lower than our proposed method (with an accuracy of $87.6 \%$ ). It should be noted that $\mathrm{Li}$ el al. obtained the best sensitivity since their work is based on sensitivity optimization, while other works have focused on optimizing total classification accuracy (Li et al. 2018). Table 7 lists the relevant pre-recording classification work and performance in which the same dataset is employed. It is noteworthy that, as we mentioned above, using traditional measurements to evaluate performance is not very accurate due to relatively small sample size (only 35 recordings in the withheld dataset), and the best method is to take the correlation value between the experimentally determined $A H I$ and the actual $A H I$ together, but not all works provide the correlation value. Nonetheless, our proposed method, with an accuracy of $97.1 \%$, provides better or comparable performance than these works presented in the literature.

\section{Conclusions}

In this study, we developed an SA detection method based on modified LeNet-5 and adjacent ECG segments. Experimental results showed that our proposed method is useful for SA detection, and the performance of our method is better than both traditional machine learning methods and existing works. Due to the high precision requirements of clinical applications, further improvements in our proposed method will accelerate the development of ECG-based SA detection devices in clinical practice. Furthermore, since only a single-lead ECG signal is used, our proposed method can also be used to develop SA detection for home healthcare services using wearable devices. However, our proposed method has some limitations. Because the Apnea-ECG dataset is labeled in 1-minute segments, an apnea/hypopnea event could occur in the middle of two 1-minute segments and a 1-minute segment could contain more than one apnea/hypopnea event. Additionally, the dataset does not separately label hypopnea and apnea events in the provided annotation file, and all events are either obstructive or mixed (central is not included). This could mean our proposed method cannot distinguish between hypopnea and apnea, and cannot detect central events. In future research, we will include other datasets to solve the above problems.

\section{Acknowledgements}


324 The authors would like to thank Dr. Thomas for providing the data.

325

326

327

328

329

330

331

332

\section{References}

Abdel-Hamid O, Mohamed A-r, Jiang H, and Penn G. 2012. Applying convolutional neural networks concepts to hybrid NN-HMM model for speech recognition. 2012 IEEE international conference on Acoustics, speech and signal processing (ICASSP): IEEE. $p$ 4277-4280.

Azarbarzin A, and Moussavi Z. 2013. Snoring sounds variability as a signature of obstructive sleep apnea. Medical engineering \& physics 35:479-485.

Bae JH, Jung KC, Kim JW, and Kim HJ. 1998. Segmentation of touching characters using an MLP. Pattern Recognition Letters 19:701-709.

Bloch KE. 1997. Polysomnography: a systematic review. Technology and health care 5:285-305.

Chen L, Zhang X, and Song C. 2015. An automatic screening approach for obstructive sleep apnea diagnosis based on single-lead electrocardiogram. IEEE Transactions on Automation Science and Engineering 12:106-115.

De Chazal P, Heneghan C, Sheridan E, Reilly R, Nolan P, and O'Malley M. 2000. Automatic classification of sleep apnea epochs using the electrocardiogram. Computers in Cardiology 2000 Vol 27 (Cat 00CH37163): IEEE. p 745-748.

El-Sawy A, Hazem E-B, and Loey M. 2016. CNN for handwritten arabic digits recognition based on LeNet-5. International Conference on Advanced Intelligent Systems and Informatics: Springer. p 566-575.

Goldberger AL, Amaral LA, Glass L, Hausdorff JM, Ivanov PC, Mark RG, Mietus JE, Moody GB, Peng C-K, and Stanley HE. 2000. PhysioBank, PhysioToolkit, and PhysioNet: components of a new research resource for complex physiologic signals. Circulation 101:e215-e220.

Hamilton PS. 2002. Open source ECG analysis software documentation. Computers in cardiology 2002:101-104.

Hornero R, Álvarez D, Abásolo D, del Campo F, and Zamarron C. 2007. Utility of approximate entropy from overnight pulse oximetry data in the diagnosis of the obstructive sleep apnea syndrome. IEEE Transactions on Biomedical Engineering 54:107-113.

Kang M, Ji K, Leng X, Xing X, and Zou H. 2017. Synthetic aperture radar target recognition with feature fusion based on a stacked autoencoder. Sensors 17:192.

Khandoker AH, Palaniswami M, and Karmakar CK. 2009. Support vector machines for automated recognition of obstructive sleep apnea syndrome from ECG recordings. IEEE transactions on information technology in biomedicine 13:37-48.

PeerJ reviewing PDF | (2019:04:36650:2:1:NEW 19 Aug 2019) 
358

359

360

361

362

363

364

365

366

367

368

369

370

371

372

373

374

375

376

377

378

379

380

381

382

383

384

385

386

387

388

389

390

391

392

393

Kiranyaz S, Ince T, and Gabbouj M. 2015. Real-time patient-specific ECG classification by 1-D convolutional neural networks. IEEE Transactions on Biomedical Engineering 63:664675.

Kwon Y-H, Shin S-B, and Kim S-D. 2018. Electroencephalography based fusion two-dimensional (2D)-convolution neural networks (CNN) model for emotion recognition system. Sensors 18:1383.

LeCun Y. 2015. LeNet-5, convolutional neural networks. URL: http://yann lecun com/exdb/lenet 20.

Li K, Pan W, Li Y, Jiang Q, and Liu G. 2018. A method to detect sleep apnea based on deep neural network and hidden markov model using single-lead ECG signal. Neurocomputing 294:94101.

Ludermir TB, Yamazaki A, and Zanchettin C. 2006. An optimization methodology for neural network weights and architectures. IEEE Transactions on Neural Networks 17:1452-1459.

Ma M, Gao Z, Wu J, Chen Y, and Zheng X. 2018. A Smile Detection Method Based on Improved LeNet-5 and Support Vector Machine. 2018 IEEE SmartWorld, Ubiquitous Intelligence \& Computing, Advanced \& Trusted Computing, Scalable Computing \& Communications, Cloud \& Big Data Computing, Internet of People and Smart City Innovation (SmartWorld/SCALCOM/UIC/ATC/CBDCom/IOP/SCI): IEEE. p 446-451.

Maier C, Bauch M, and Dickhaus H. 2000. Recognition and quantification of sleep apnea by analysis of heart rate variability parameters. Computers in Cardiology 2000 Vol 27 (Cat 00CH37163): IEEE. p 741-744.

Marcus CL, Brooks LJ, Ward SD, Draper KA, Gozal D, Halbower AC, Jones J, Lehmann C, Schechter MS, and Sheldon S. 2012. Diagnosis and management of childhood obstructive sleep apnea syndrome. Pediatrics 130:e714-e755.

Matsugu M, Mori K, Mitari Y, and Kaneda Y. 2003. Subject independent facial expression recognition with robust face detection using a convolutional neural network. Neural Networks 16:555-559.

Mostafa SS, Morgado-Dias F, and Ravelo-García AG. 2018. Comparison of SFS and mRMR for oximetry feature selection in obstructive sleep apnea detection. Neural Computing and Applications:1-21.

Palaz D, and Collobert R. 2015. Analysis of cnn-based speech recognition system using raw speech as input. Idiap.

Penzel T, Kantelhardt JW, Grote L, Peter J-H, and Bunde A. 2003. Comparison of detrended fluctuation analysis and spectral analysis for heart rate variability in sleep and sleep apnea. IEEE Transactions on Biomedical Engineering 50:1143-1151.

Penzel T, Moody GB, Mark RG, Goldberger AL, and Peter JH. 2000. The apnea-ECG database. 
394

395

396

397

398

399

400

401

402

403

404

405

406

407

408

409

410

411

412

413

414

415

416

417

418

419

420

421

422

423

424

425

426

427

428

429

Computers in Cardiology 2000 Vol 27 (Cat 00CH37163): IEEE. p 255-258.

Peppard PE, Young T, Barnet JH, Palta M, Hagen EW, and Hla KM. 2013. Increased prevalence of sleep-disordered breathing in adults. American journal of epidemiology 177:1006-1014.

Punjabi NM. 2008. The epidemiology of adult obstructive sleep apnea. Proceedings of the American Thoracic Society 5:136-143.

Sedighi B, Palit I, Hu XS, Nahas J, and Niemier M. 2015. A CNN-inspired mixed signal processor based on tunnel transistors. 2015 Design, Automation \& Test in Europe Conference \& Exhibition (DATE): IEEE. p 1150-1155.

Sharif Razavian A, Azizpour H, Sullivan J, and Carlsson S. 2014. CNN features off-the-shelf: an astounding baseline for recognition. Proceedings of the IEEE conference on computer vision and pattern recognition workshops. p 806-813.

Sharma H, and Sharma K. 2016. An algorithm for sleep apnea detection from single-lead ECG using Hermite basis functions. Computers in biology and medicine 77:116-124.

Song C, Liu K, Zhang X, Chen L, and Xian X. 2016. An obstructive sleep apnea detection approach using a discriminative hidden Markov model from ECG signals. IEEE Transactions on Biomedical Engineering 63:1532-1542.

Srivastava N, Hinton G, Krizhevsky A, Sutskever I, and Salakhutdinov R. 2014. Dropout: a simple way to prevent neural networks from overfitting. The Journal of Machine Learning Research 15:1929-1958.

Varon C, Caicedo A, Testelmans D, Buyse B, and Van Huffel S. 2015. A novel algorithm for the automatic detection of sleep apnea from single-lead ECG. IEEE Transactions on Biomedical Engineering 62:2269-2278.

Wei Y, Xia W, Lin M, Huang J, Ni B, Dong J, Zhao Y, and Yan S. 2016. HCP: A flexible CNN framework for multi-label image classification. IEEE transactions on pattern analysis and machine intelligence 38:1901-1907.

Wen L, Li X, Gao L, and Zhang Y. 2018. A new convolutional neural network-based data-driven fault diagnosis method. IEEE Transactions on Industrial Electronics 65:5990-5998.

Xie B, and Minn H. 2012. Real-time sleep apnea detection by classifier combination. IEEE transactions on information technology in biomedicine 16:469-477.

Yadollahi A, and Moussavi Z. 2009. Acoustic obstructive sleep apnea detection. 2009 annual international conference of the IEEE engineering in medicine and biology society: IEEE. p 7110-7113.

Yin W, Kann K, Yu M, and Schütze H. 2017. Comparative study of cnn and rnn for natural language processing. arXiv preprint arXiv:170201923.

Young T, Peppard P, Palta M, Hla KM, Finn L, Morgan B, and Skatrud J. 1997. Population-based study of sleep-disordered breathing as a risk factor for hypertension. Archives of internal

Peer) reviewing PDF | (2019:04:36650:2:1:NEW 19 Aug 2019) 
$430 \quad$ medicine 157:1746-1752.

431 Zeiler MD, and Fergus R. 2014. Visualizing and understanding convolutional networks. European 432 conference on computer vision: Springer. p 818-833.

433

434 
Table $\mathbf{1}$ (on next page)

Details of our modified LeNet-5 convolutional neural network 


\begin{tabular}{llll}
\hline Layer & Parameter & Output Shape & Number $^{\mathbf{a}}$ \\
\hline Input & - & $($ None, 900, 2) & 0 \\
Conv1 & $32 \times 5 \times 2$, stride 2, pad 0 & $($ None, 448, 32) & 352 \\
Max pooling2 & 3, stride 3, pad 0 & $($ None, 149, 32) & 0 \\
Conv3 & 64×5×2, stride 2, pad 0 & (None, 73, 64) & 10304 \\
Max pooling4 & 3, stride 3, pad 0 & (None, 24, 64) & 0 \\
Dropout5 & 0.8 rate & (None, 24, 64) & 0 \\
FC6 & 32, relu & (None, 32) & 49184 \\
Output & 2, softmax & (None, 2) & 66 \\
\hline
\end{tabular}

a The number of parameters generated by the corresponding operation. 
Table 2 (on next page)

Feature set extracted based on previous studies 


\begin{tabular}{|c|c|c|c|}
\hline \multirow[t]{2}{*}{ Name } & \multicolumn{2}{|c|}{ Derived from } & \multirow[t]{2}{*}{ Details } \\
\hline & $\mathbf{R R}^{\mathbf{a}}$ & Ampl ${ }^{b}$ & \\
\hline RMSSD & $\times$ & & $\begin{array}{l}\text { Square root of the average of the squared difference } \\
\text { between adjacent RR intervals. }\end{array}$ \\
\hline SDNN & $x$ & & $\begin{array}{l}\text { Standard deviation of the difference between adjacent } \\
\text { RR intervals. }\end{array}$ \\
\hline NN50 & $\times$ & & Number of adjacent RR intervals exceeds 50 ms. \\
\hline pNN50 & $\times$ & & NN50 divides by the number of RR intervals. \\
\hline Mean RR & $x$ & & Mean of RR intervals. \\
\hline Mean HR & $\times$ & & $\begin{array}{l}\text { Mean of heart rate (HR), which is derived from } R R \\
\text { intervals. }\end{array}$ \\
\hline Normalized VLF & $\times$ & $\times$ & $\begin{array}{l}\text { Normalized very low frequency (VLF) component of the } \\
\text { corresponding signal. }\end{array}$ \\
\hline Normalized LF & $x$ & $\times$ & $\begin{array}{l}\text { Normalized Low frequency (LF) component of the } \\
\text { corresponding signal. }\end{array}$ \\
\hline Normalized HF & $x$ & $\times$ & $\begin{array}{l}\text { Normalized high frequency (HF) component of the } \\
\text { corresponding signal. }\end{array}$ \\
\hline $\mathbf{L F} / \mathbf{H F}$ & $\times$ & $\times$ & The ratio of LF to HF of the corresponding signal. \\
\hline $\mathbf{L F} /(\mathbf{L F}+\mathbf{H F})$ & $\times$ & $\times$ & The ratio of LF to LF+HF of the corresponding signal. \\
\hline $\mathrm{HF} /(\mathbf{L F}+\mathrm{HF})$ & $\times$ & $\times$ & The ratio of HF to $\mathrm{LF}+\mathrm{HF}$ of the corresponding signal. \\
\hline
\end{tabular}




\section{Table 3 (on next page)}

The overall performance of our modified LeNet-5 and traditional machine learning methods in per-segment SA detection 


\begin{tabular}{lllll}
\hline Method & Accuracy (\%) & Sensitivity (\%) & Specificity (\%) & AUC \\
\hline SVM & 81.4 & 76.9 & 84.3 & 0.887 \\
LR & 80.8 & 75.7 & 84.0 & 0.884 \\
KNN & 77.5 & 68.1 & 83.4 & 0.826 \\
MLP & 81.1 & 71.3 & 87.2 & 0.898 \\
LeNet-5 & 87.6 & 83.1 & 90.3 & 0.950 \\
\hline
\end{tabular}

1 


\section{Table 4 (on next page)}

The overall performance of our modified LeNet-5 and traditional machine learning methods in per-recording classification 


\begin{tabular}{llllll}
\hline Method & Accuracy (\%) & Sensitivity (\%) & Specificity (\%) & AUC & Corr. $^{\text {a }}$ \\
\hline SVM & 88.6 & 100.0 & 66.7 & 0.978 & 0.852 \\
LR & 88.6 & 100.0 & 66.7 & 0.982 & 0.841 \\
KNN & 82.9 & 100.0 & 50.0 & 0.986 & 0.845 \\
MLP & 85.7 & 95.7 & 66.7 & 0.949 & 0.814 \\
LeNet-5 & 97.1 & 100.0 & 91.7 & 0.996 & 0.943 \\
\hline
\end{tabular}

1 a The correlation value between the actual $A H I$ and the experimentally determined $A H I$. 


\section{Table 5 (on next page)}

The per-segment SA detection and per-recording classification performance in the UCDDB database 


\begin{tabular}{|c|c|c|c|c|c|c|c|}
\hline \multirow[b]{2}{*}{ Classifier } & \multicolumn{3}{|c|}{ Per-segment } & \multicolumn{4}{|c|}{ Per-recording } \\
\hline & Accuracy (\%) & Sensitivity (\%) & Specificity (\%) & Accuracy (\%) & Sensitivity (\%) & Specificity (\%) & $\begin{array}{l}\text { Corr } \\
\text {. }\end{array}$ \\
\hline SVM & 70.6 & 32.7 & 83.3 & 92.3 & 100.0 & 50.0 & 0.251 \\
\hline $\mathbf{L R}$ & 69.6 & 34.7 & 81.3 & 84.6 & 90.9 & 50.0 & 0.107 \\
\hline KNN & 66.1 & 38.1 & 75.4 & 84.6 & 100.0 & 0.0 & 0.373 \\
\hline MLP & 67.2 & 38.5 & 76.8 & 92.3 & 100.0 & 50.0 & 0.263 \\
\hline LeNet-5 & 71.8 & 26.6 & 86.9 & 92.3 & 90.9 & 100.0 & 0.624 \\
\hline
\end{tabular}


Table 6(on next page)

Comparison between the per-segment SA detection performance of our modified LeNet-5 and existing works 


\begin{tabular}{|c|c|c|c|c|c|}
\hline Reference & Features & Classifier & $\begin{array}{l}\text { Accuracy } \\
(\%)\end{array}$ & $\begin{array}{l}\text { Sensitivit } \\
\text { y (\%) }\end{array}$ & $\begin{array}{l}\text { Specificity } \\
(\%)\end{array}$ \\
\hline (Varon et al. 2015) & $\begin{array}{l}\text { Feature } \\
\text { Engineering }\end{array}$ & LS-SVM & 84.7 & 84.7 & 84.7 \\
\hline (Song et al. 2016) & $\begin{array}{l}\text { Feature } \\
\text { Engineering }\end{array}$ & $\begin{array}{l}\text { HMM- } \\
\text { SVM }\end{array}$ & 86.2 & 82.6 & 88.4 \\
\hline (Sharma \& Sharma 2016) & $\begin{array}{l}\text { Feature } \\
\text { Engineering }\end{array}$ & LS-SVM & 83.4 & 79.5 & 88.4 \\
\hline (Li et al. 2018) & $\begin{array}{l}\text { Auto } \\
\text { encoder }\end{array}$ & $\begin{array}{l}\text { Decision } \\
\text { fusion }\end{array}$ & 83.8 & 88.9 & 88.4 \\
\hline Our study & $\mathrm{CNN}$ & LeNet-5 & 87.6 & 83.1 & 90.3 \\
\hline
\end{tabular}




\section{Table 7 (on next page)}

Comparison between the per-recording classification performance of our modified LeNet-5 and existing works 


\begin{tabular}{|c|c|c|c|c|c|}
\hline Reference & Classifier & $\begin{array}{l}\text { Accuracy } \\
(\%)\end{array}$ & $\begin{array}{l}\text { Sensitivity } \\
(\%)\end{array}$ & $\begin{array}{l}\text { Specificity } \\
(\%)\end{array}$ & Corr. \\
\hline (Morillo \& Gross 2013) & PNN & 93.8 & 92.4 & 95.9 & - \\
\hline (Sharma \& Sharma 2016) & LS-SVM & 97.1 & 95.8 & 100 & 0.841 \\
\hline (Song et al. 2016) & HMM-SVM & 97.1 & 95.8 & 100 & 0.860 \\
\hline (Alvarez et al. 2010) & LR & 89.7 & 92.0 & 85.4 & - \\
\hline (Li et al. 2018) & $\begin{array}{l}\text { Decision } \\
\text { fusion }\end{array}$ & 100 & 100 & 100 & - \\
\hline Our study & LeNe-5t & 97.1 & 100.0 & 91.7 & 0.943 \\
\hline
\end{tabular}

1 


\section{Figure 1}

PhysioNet Apnea-ECG dataset preprocessing scheme. Note: In this study, the labeled segment and its surrounding \pm 2 segments of the ECG signal (five 1-minute segments in total) was extracted as a whole for processing. 


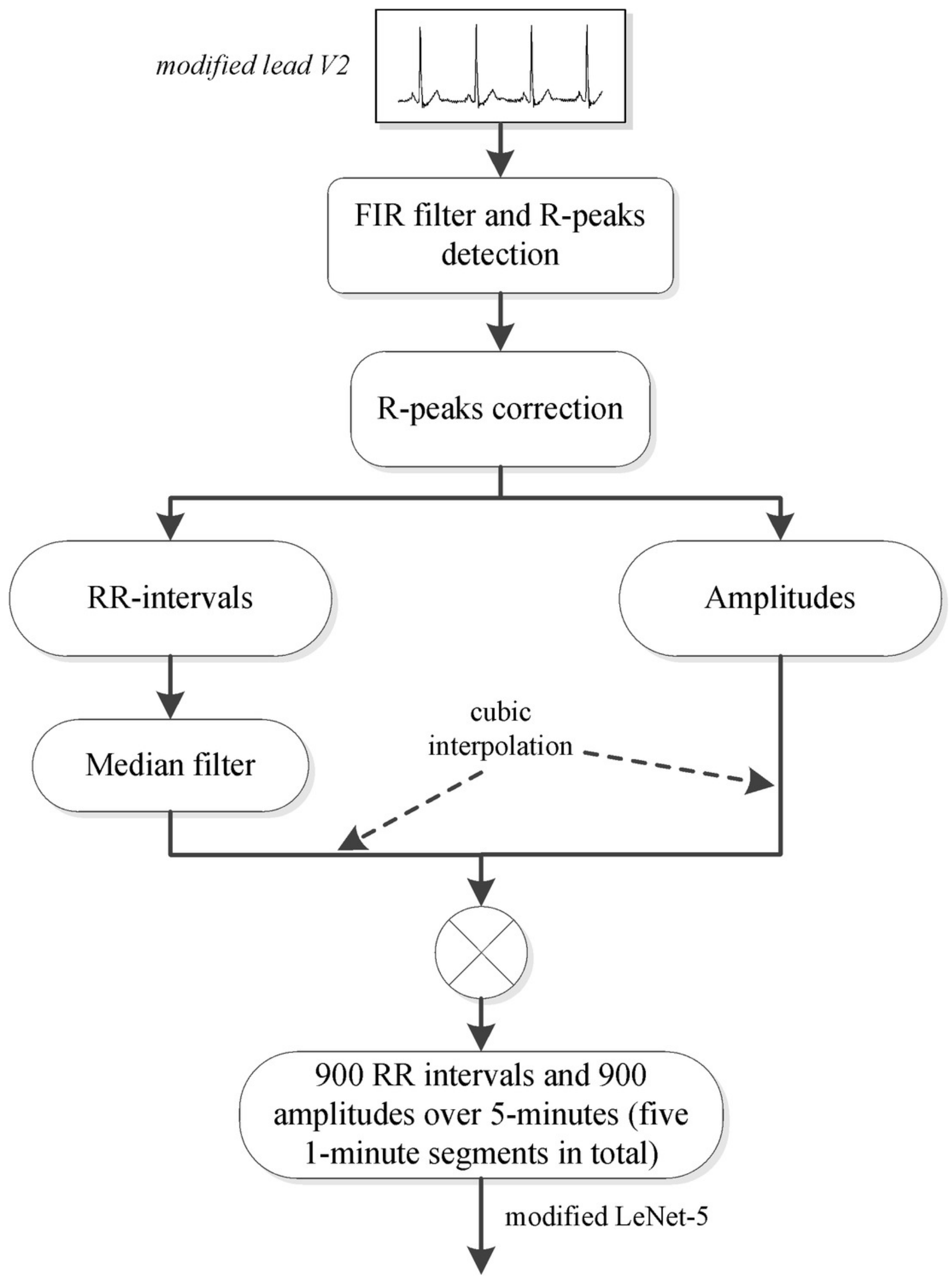


Figure 2

Architecture of our modified LeNet-5. It can be seen as a combination of convolutional neural networks (CNN) for feature extraction and full connection (FC, also known as MLP) as classifier

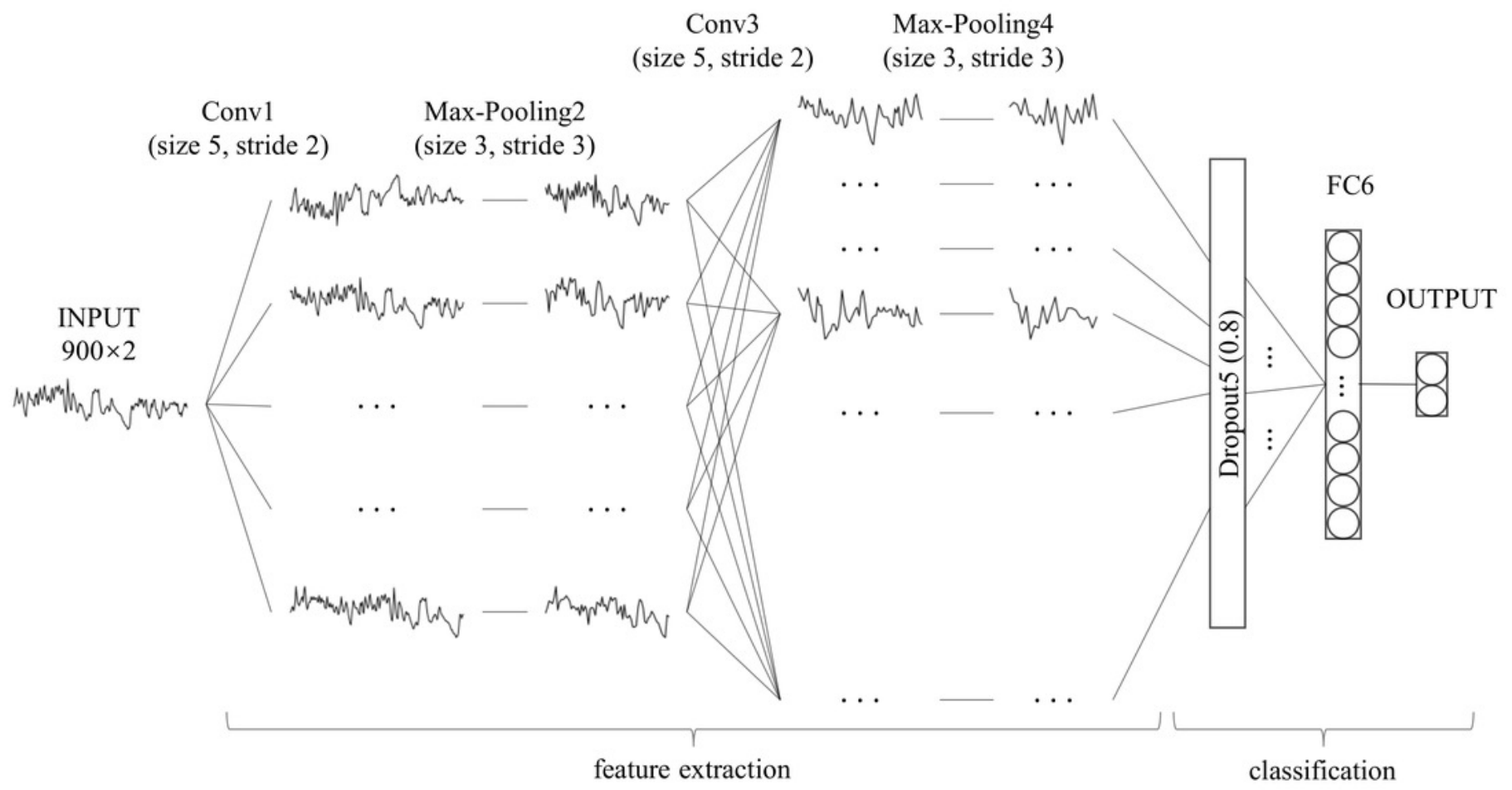


Figure 3

Comparison of ROC curves of our modified LeNet-5 and MLP in per-segment SA detection

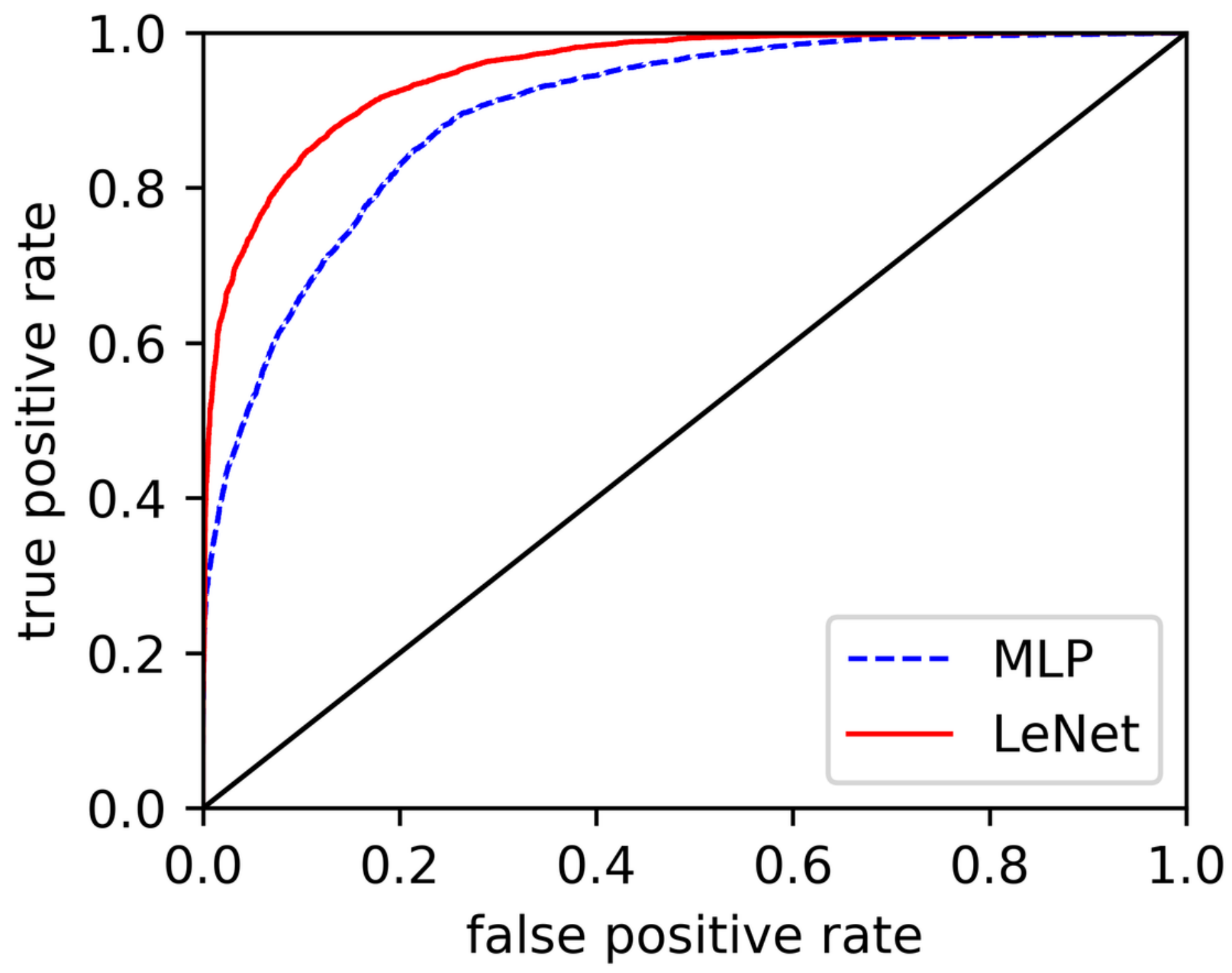


Figure 4

Comparison of the per-segment detection accuracy of five classifiers calculated on 10 different test groups

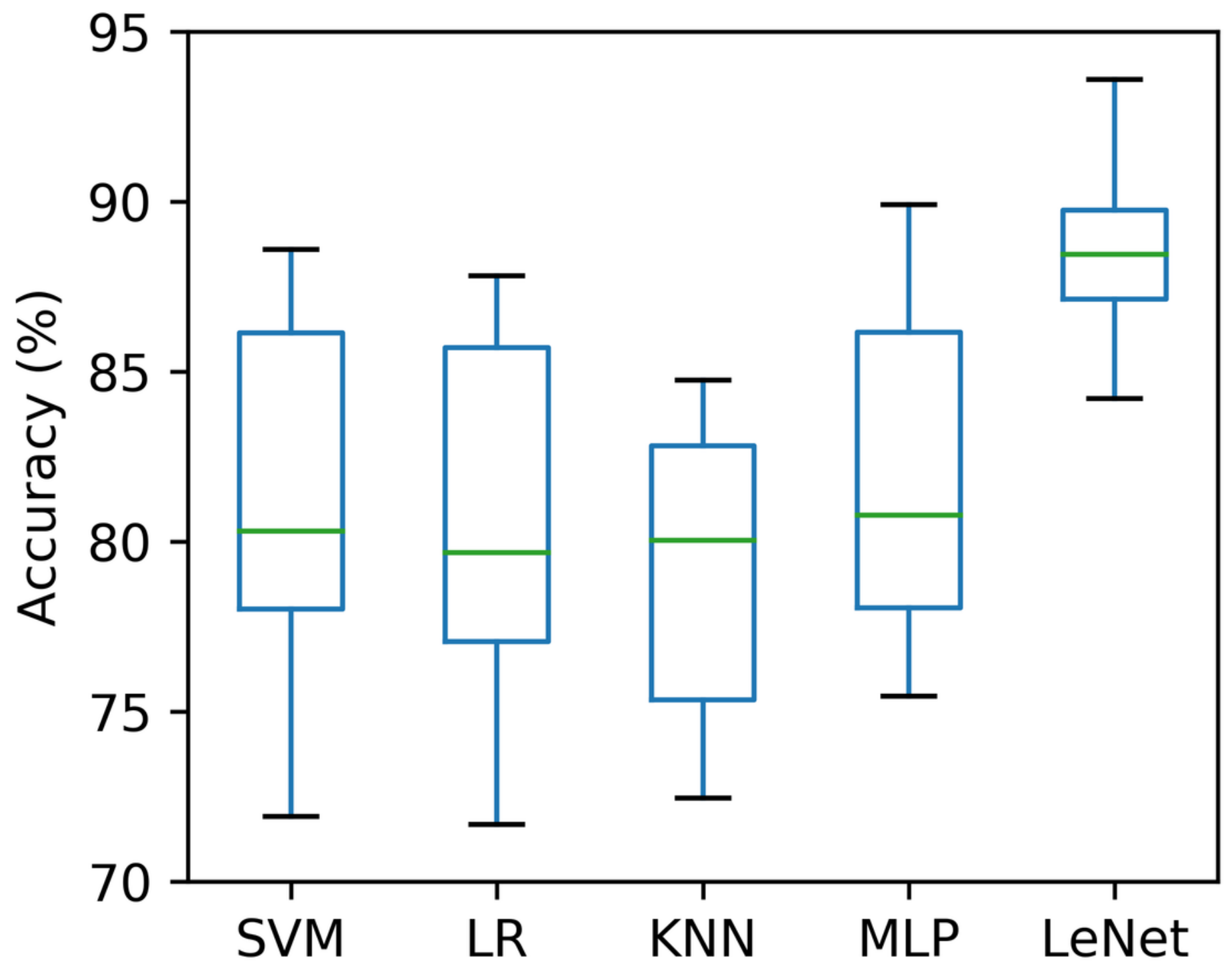

